\title{
The Cultivation of Hurdle Athletes' Psychological Ability
}

\author{
Guo $\mathrm{Lu}^{1, \mathrm{a}}$, Xianhong $\mathrm{Lin}^{2, \mathrm{a}}$, Dan $\mathrm{Li}^{3, \mathrm{a}}$ and Xiaoyi He $\mathrm{He}^{4, \mathrm{a}}$ \\ ${ }^{1}$ Jilin Agricultural University, Xincheng Street 2888, Changchun, Jilin Province 130118, PR China \\ 2,3,4 Jilin Sport University,2476 Ziyou Road, Changchun City, Jilin Province 130118, PR China \\ a360282476@qq.com
}

Keywords: Hurdle; Athletic performance; Psychological quality college students

\begin{abstract}
In recent years, with the continuous improvement of competitive sports in China, more and more scientific training methods and methods into the sports training. In our hurdle training, coaches are often concerned with problems in the field of technology and their solutions, and to explore the improvement of technology at the same time to improve the necessary physical fitness, increase the physiological end of the limit, play the nerve and muscle Between the more effective cooperation and so on, and often ignore the psychological quality of training in this area. Sports experts in the world through practice to prove that the cultivation of psychological quality is an indispensable part of the entire training system, it can complement and make the body, technical training more rationalized, so many factors of psychological quality determines the level of hurdler athletes play, It is necessary to once again demonstrate the importance of hurdle athletes psychological quality.
\end{abstract}

\section{Preface}

Hurdle movement first appeared in the British nineteenth century, the use of the general barrier to do obstacles. And as a form of human movement across obstacles is human in the long-term production and struggle with nature in the formation of a basic life skills. As a hurdle of track and field sports, is the basic skills across the obstacles, the development evolved. The famous athlete Liu Xiang in the hurdle made in the breakthrough results, the hurdle of our country to a peak. Understanding the history and movement of hurdles is a prerequisite for the development of hurdles and the training of their project athletes. And the cultivation of athletes' psychological quality is to meet the characteristics of this movement. In the hurdle training and competition, there are many factors that affect the performance of the game, but the psychological quality is the key to training and competition. Psychological quality often play an important role in the game, the size of the game, the opponent's level, weather conditions, athletes bear the task, etc., these will be psychological to the athletes caused some pressure. In this regard, in the daily training, we should focus on the cultivation of athletes psychological quality, so as to achieve better athletic performance to lay the foundation.

\section{Research Methods}

Literature Method. Through the knowledge of the network of athletes about the psychological quality of the professional literature more than 30 articles, and finishing the summary, to provide theoretical reference for this article.

Expert Interview. Through the visit to more hurdles coaches in Jilin Province, learn more about the psychological factors that exist in the training or competition of more hurdlers.

\section{Results and Analysis}

The Characteristics of Hurdle Movement. Hurdle Race is a kind of personal nature with a strong direct competitive project, the official race with men $110 \mathrm{~m}$ hurdles, women's $100 \mathrm{~m}$ hurdles 
and male and female $400 \mathrm{~m}$ hurdles, athletes in the game must use a fixed number of steps to finish a fixed distance between the columns, Across a fixed height of the shelf, there are significant special features. From the activities of the structure point of view, both the activities of the activities of the project structure, such as: column before the run, run between the column, the end run, so that single foot support and vacated alternating periodic muscle activity, but also has a cross, , The next column when the corresponding muscle joints were asymmetric activities, in general, is a run from the cross, from cross to run to change the structure of the activities of the coordinated activities of the process. From the activities of the conditions, the men's $110 \mathrm{~m}$ hurdles and women's $100 \mathrm{~m}$ bar is the ultimate strength of movement, $400 \mathrm{~m}$ bar sub-limit strength movement, are in the provisions of the venue and a series of competition rules under the conditions of competition with the opponent directly, its activities The result is the fastest speed to finish the whole process as a sign.

The Importance of the Psychological Quality of Hurdle Athletes. In today's world of sports research and technical level of continuous improvement and innovation in the fierce sports competition between athletes is not only a physical, skill contest, but also a psychological contest, because in the fierce competition athletes not only to The greater the psychological load, the more intense the psychological load, the two sides in the skills, physical fitness, tactical ability, training level and strength of the case, the psychological quality of the game will often play a decisive role in the outcome. Hurdle movement is a relatively complex technology, a strong sense of rhythm and a certain degree of difficulty of the sport, it not only requires athletes must have a comprehensive physical and technical, tactical level, but also requires athletes with good psychological quality. In the athlete's game or training, due to the process of running a certain number of columns, the height of the column, the number of provisions of the distance between the bar and other conditions have been presented in front of the athletes, athletes to complete any a kind of technical and tactical action must be subject to their own physical and psychological constraints and other factors such as constraints and influence, must be with the emotions, will and personality and other characteristics associated with, therefore, good psychological quality will help athletes to play the technology.

The Cultivation of Hurdle Athletes' Psychological Quality. Modern sports competition shows that athletes to participate in the game, not only to consume a lot of physical strength, but also consume a lot of psychological energy. Psychological quality as a new means of training into the field of sports training, and the traditional physical training, technical training, tactical training together, constitute a complete system of sports training. In the practice of sports, the cultivation of psychological quality has achieved remarkable results. Sports competition, athletes in order to achieve excellent athletic performance, not only to carry out physical and technical preparation, but also to carry out all kinds of psychological preparation. Good pre-match psychological state, athletes can give full play to their physical, technical and tactical level, the formation of the best psychological state of the game, is to go all out to participate in training and competition, and achieved excellent results basis. Hurdle movement is a high quality requirements of the project, the technical requirements of the project hurdler athletes psychological quality training, to have a purpose, a planned athlete heart process and personality, psychological characteristics of the process of exertions, but also to enable athletes to adjust and control their own psychological State and then adjust and control their own movement of the process.

The Cultivation of General Psychological Quality of Hurdle Athletes. General psychological training is "long-term psychological training, intangible psychological training", the main task is to train athletes a good motivation and the correct attitude of training. In the course of training, the psychological quality of the athletes should be cultivated. First, the psychological quality and personality characteristics of the athletes should be diagnosed. According to the individual characteristics and psychological status of the athletes, the training should be arranged differently. In the commonly used general teaching training, usually use the intuitive teaching method to explain the demonstration so that athletes to establish a certain correct concept of sports technology, and then enter the individual training, from simple to complex and repeated contact and training. With the athletes age, psychological characteristics and physiological characteristics and physical quality of the continuous improvement in the coaches fully demonstrated and explained on the basis 
of the use of technical pictures, technical videos and simple language prompts, the use of imagination and inner images, to establish a correct The technical concept.

Inspire the Right Learning Motivation. To maintain a good mental state, give full play to favorable factors, continue to overcome the unfavorable factors in order to obtain satisfactory training results. This requires the creation of good psychological conditions, training to adjust the appropriate psychological state. The so-called appropriate psychological state, the actual is to make the movement of the cerebral cortex in a suitable state of excitement. It can promote the development of differentiation inhibition, so that the excitement of the cerebral cortex can be faster concentration, the establishment of the correct conditional reflex, to speed up the formation of action skills. The process of training and regulating the mental state also includes the process of ideological education, which requires the coaches in the process of sports training in addition to stimulate the athletes initiative, enthusiasm, but also pay attention to cultivate them to overcome the difficulties of the will quality, to overcome the psychological Fear, so as to achieve the purpose of improving the effect of practice.

Strengthen the Sense of Hurdle. The establishment of the sense of hurdle has a close relationship with the improvement of psychological function. To guide the athletes to practice the basic skills of the column, from the hurdle shape to the combination of technology to continuously strengthen the process of time and space perception, and gradually establish a "sense of hurdle" to improve the athletes to accurately feel and control the hurdles and the whole process of the whole column Ability to overcome the fear of the column and the emergence of the separation of people column jumping action.

On the Cultivation of Hurdle Athletes' Psychological Quality. The biggest obstacle to the athlete's psychology is the adverse reaction that occurs when the game is produced. This psychological adjustment should be carried out before the game in order to meet the competitive environment in a good state in the game with good results. So that psychological training is the game to win the secret weapon.

Psychological training, in a broad sense, is consciously on the psychological process of athletes and personality psychological characteristics of the impact of athletes for good results for all kinds of psychological preparation; in the narrow sense, is to enable athletes to adjust their mental state Various methods, to better participate in sports training and complete complex tasks of the game. Athlete's psychological training is a long and long process, never done overnight, even with the athletes throughout the sporting career. West Germany's sports psychologist Riddle said: "The psychological preparation of the competition is a long-term learning process. In this process, in addition to success must also withstand failure and frustration, and finally make the athletes become an independent, stable - that is able to resist a variety of interference factors. "It can be seen that the main purpose of psychological training is to enable athletes to adapt to the changing conditions of the game and the game environment and a variety of external factors, in the game encounter a variety of situations can maintain a calm psychological, accurate and flexible use of technology Get good grades.

Athletes of the level of psychological training, will directly affect the athletes of their own body and technology, tactical regulation, or play a positive role in promoting, or play a negative obstruction. The athlete's psychological training level has a great influence on the performance of his game. Psychologists believe that in a high level of sports competitions in the psychological quality of the game results of $70 \%$ to $80 \%$ or even $90 \%$. This is what people often say "more than psychological". In practice, many potential athletes can not create excellent results, the reason is because they usually ignore the cultivation of psychological quality, so at the crucial moment fall short. The psychological quality of athletes are based on the quality of innate, through the day after the training and training, especially through the training of the training and the accumulation of experience gradually formed. So pay attention to the following aspects of training:

Strengthen Pre-competition Psychological Control. Pre-competition status refers to changes in the reflexivity of the body's performance due to the understanding of the competition and the resulting emotional experience before the start of the competition. These changes include changes 
in the excitability of the nerve center; Changes in pulse, blood pressure, body temperature and sweat gland activity, changes in blood glucose levels, these changes have a significant impact on the upcoming game. According to the study of exercise time, athletes have four pre-game state: before the game too excited state; pre-game indifferent state; combat prepared state; blind confidence before the state. Therefore, the athletes from the game before you can listen to cheerful music, think of their own good wishes, transfer the tension, the appropriate relaxation of their own feelings.

Cultivate the Special Psychological Quality of Sports Competition. To carry out sports competitions, first of all to the usual training of psychological quality into the needs of the competition. Sports competition in the exercise intensity, technical action proficiency and body load, are different from the usual training level, which requires the psychological quality of the preparatory training. To promote the adaptation of the game environment, any competition environment conditions, are not equivalent to the usual sports training. Many athletes are unable to adapt to the situation on the game and lead to defeat. This failure from the objective point of view, it seems that the body, the technology does not meet, but from the microscopic point of view, often lead to the reasons for the failure is actually in the psychological aspects. Coaches should be based on the actual situation, in conditions permitting the athletes to participate in large-scale competition, or training in foreign countries, to adapt to the various contest environment, create a good external environment, to overcome the psychological tension before the game.

Dilute Ranking of Competition, Reduce the Psychological Burden. Ranking and other hard targets, for athletes can be a driving force, but in the case of heavy pressure, this indicator pressure will often give athletes unnecessary psychological burden, want to win fear of losing, in the game suffer. For such athletes, coaches should try to avoid the athletes before the game to issue a rigid task of the task, to reduce the psychological burden of athletes, play the best level

Cultivate the Ability to Adapt to the Environment. Cultivate the appropriate internal environment, improve their own psychological quality, and constantly improve themselves. Emphasizing the athletes themselves, improve the psychological quality of athletes. Athletes as the protagonist of the game, is always the focus of the game. Therefore, for athletes, should continue to strengthen learning, with a wealth of cultural heritage and publicity personality to infect the referee and the audience on the game, so that in any occasion or any situation, can be calm, do win Arrogance, failure is not discouraged, able to face reality, objective evaluation of their own. Not because of a moment of failure or success and change their mentality, always with the correct game motive, according to the specific circumstances and coaches designated appropriate goals, the correct treatment of the game.

Make Good Self Hints. Self-hinting, forming a good psychological state of the game. Self-suggestion is a process of self-stimulation. It can change in behavior and subjective experience to produce an effect, causing some psychological, physiological changes in the corresponding. Before the game, regardless of what kind of game, the opponent level, should be self-appropriate control. Among them, self-suggestion is a very good means of control. Through self-suggestion, to maintain a good mental state, a better solution to the recovery of mental energy problems, put their place, establish a strong confidence to establish long-term goals, to better match. So that athletes in the game have a good psychological preparation, play a better level.

\section{Conclusion and Suggestion}

Conclusion. Psychological quality is a special training, his good or bad determines the athletes play. At present, both the world sports or our sports, hurdles are athletes are concerned about the popular projects, hurdle training methods are constantly evolving in a number of advanced breakthroughs. For this complex technical sport, the training of athletes psychological quality is very important. Therefore, the athletes must be based on the characteristics of the hurdle athletes and vary from person to person, continue to explore, constantly sum up, with the most appropriate psychological quality of the nesting method of each athlete's physical fitness, psychological ability, technical ability to fully convert to the most good competitive state, all of these must rely on psychological quality to complete. 
Suggestion. Improve the quality of coaches themselves. The quality of the coaches has a great influence on the athletes. In the sports training, should be aimed at the coaches of their own quality and cultural quality to cultivate and improve the coaches in the ideological progress, so as to use more advanced means to guide the training.

The training of athletes' psychological quality refers to the trainer's training plan. From a number of points of view can be seen the importance of psychological quality of the hurdler, which reflects the cultivation of psychological quality and competitive training as important, so the psychological quality should be the same with the training of athletes, the same should be included in the coaches training program Among them, for different athletes to develop different training programs.

\section{References}

[1] Y.C Dong, et al. Psychological skills training in.110 meters hurdles [J]. Journal of Hebei Energy Institute of Vocation and Technology, 2008, (2): 86-87.

[2] W.S Wang, G.Q Dai, Discussion on the psychological training of young athletes in track and field [J]. Journal of Harbin Sport University.2009; 23 (88); 103-105.

[3] J Wang, etc Talking about the psychological characteristics and training of high level hurdler athletes [J].Journal of Physical Education Institute of Shanxi Teachers University, 2010; 19 (3): 77-79.

[4] M.L Ji, et al. [J].Journal of Nanjing Sport Institute, 2009; 3 (4): $45-47$.

[5] W.J Du, Liu Xiang's success on the sports school juvenile hurdle training enlightenment [J]. Journal.

[6] J.H Yang, Analysis of hurdle training psychological barriers [J] .Journal of Zhengzhou College of Animal Husbandry Engineering, 2008; (2): 75-76.

[7] J.M Wang, B.H Wang and X Liu, 110 meters hurdle athletic performance thinking: training methods and psychological advantages [J].Chinese Journal of Clinical Rehabilitation, 2009; (5): $149-151$. 